\title{
Global Attractor of Thermoelastic Coupled Beam Equations with Structural Damping
}

\author{
Peirong Shi, ${ }^{1}$ Danxia Wang, ${ }^{2}$ and Weiyi Chen ${ }^{1}$ \\ ${ }^{1}$ Mechanics College, Taiyuan University of Technology, Taiyuan 030024, China \\ ${ }^{2}$ Department of Mathematics, Taiyuan University of Technology, Taiyuan 030024, China \\ Correspondence should be addressed to Danxia Wang; danxia.wang@163.com \\ Received 17 November 2016; Accepted 21 December 2016; Published 22 March 2017 \\ Academic Editor: Rahmat Ellahi
}

Copyright (c) 2017 Peirong Shi et al. This is an open access article distributed under the Creative Commons Attribution License, which permits unrestricted use, distribution, and reproduction in any medium, provided the original work is properly cited.

In this paper, we study the existence of a global attractor for a class of $n$-dimension thermoelastic coupled beam equations with structural damping $u_{t t}+\Delta^{2} u+\Delta^{2} u_{t}-\left[\sigma\left(\int_{\Omega}^{0}(\nabla u)^{2} d x\right)+\phi\left(\int_{\Omega} \nabla u \nabla u_{t} d x\right)\right] \Delta u+f_{1}(u)+g\left(u_{t}\right)+v \Delta \theta=q(x)$, in $\Omega \times R^{+}$, and $\theta_{t}-\Delta \theta+$ $f_{2}(\theta)-v \Delta u_{t}=0$. Here $\Omega$ is a bounded domain of $R^{N}$, and $\sigma(\cdot)$ and $\phi(\cdot)$ are both continuous nonnegative nonlinear real functions and $q$ is a static load. The source terms $f_{1}(u)$ and $f_{2}(\theta)$ and nonlinear external damping $g\left(u_{t}\right)$ are essentially $|u|^{\rho} u,|\theta|^{\rho} \theta$, and $\left|u_{t}\right|^{r} u_{t}$ respectively.

\section{Introduction}

This problem is based on the equation

$$
u_{t t}+u_{x x x x}-\left(\alpha+\beta \int_{0}^{L} u_{x}^{2} d x\right) u_{x x}=0,
$$

which was proposed by Woinowsky-Krieger [1] as a model for vibrating beam with hinged ends.

Without thermal effects, Ball [2] studied the initialboundary value problem of more general beam equation

$$
u_{t t}+u_{x x x x}-M\left(\int_{0}^{L} u_{x}^{2} d x\right) u_{x x}=0
$$

subjected to homogeneous boundary condition. Ma and Narciso [3] proved the existence of global solutions and the existence of a global attractor for the Kirchhoff-type beam equation

$$
\begin{aligned}
u_{t t} & +\Delta^{2} u-M\left(\int_{\Omega}(\nabla u)^{2} d x\right) \Delta u+f(u)+g\left(u_{t}\right) \\
& =h(x),
\end{aligned}
$$

without structural damping, subjected to the conditions

$$
u=\frac{\partial u}{\partial v}=0 \quad \text { on } \partial \Omega \times R^{+} .
$$

In fact, the plate equations without thermal effects were studied by several authors; we quote, for instance, [4-8].

In the following we also make some comments about previous works for the long-time dynamics of thermoelastic coupled beam system with thermal effects.

Giorgi et al. [9] studied a class of one-dimensional thermoelastic coupled beam equations

$$
\begin{aligned}
& u_{t t}+\Delta^{2} u-\left(\beta+\|\nabla u\|_{L^{2}(0, l)}^{2}\right) \Delta u-\Delta u_{t t}+f(u)+\Delta \theta \\
& \quad=f,
\end{aligned}
$$

and gave the existence and uniqueness of global weak solution and the existence of global attractor under Dirichlet boundary conditions. Barbosa and $\mathrm{Ma}$ [10] studied the long-time behavior for a class of two-dimension thermoelastic coupled beam equation

$$
\begin{aligned}
u_{t t} & +\Delta^{2} u-M\left(\int_{\Omega}|\nabla u|^{2} d x\right) \Delta u-\Delta u_{t t}+f(u)+v \Delta \theta \\
& =h(x),
\end{aligned}
$$


subjected to the conditions

$$
\begin{aligned}
& u=\Delta u=0, \\
& \theta=0 .
\end{aligned}
$$

In addition, we also refer the reader to [11-15] and the references therein.

A mathematical problem is the nonlinear $n$-dimension thermoelastic coupled beam equations with structural damping which arise from the model of the nonlinear vibration beam with Fourier thermal conduction law:

$$
\begin{aligned}
& u_{t t}+\Delta^{2} u+\Delta^{2} u_{t} \\
&- {\left[\sigma\left(\int_{\Omega}(\nabla u)^{2} d x\right)+\phi\left(\int_{\Omega} \nabla u \nabla u_{t} d x\right)\right] \Delta u } \\
&+f_{1}(u)+g\left(u_{t}\right)+v \Delta \theta=q(x), \quad \text { in } \Omega \times R^{+}, \\
& \theta_{t}-\Delta \theta+f_{2}(\theta)-v \Delta u_{t}=0
\end{aligned}
$$

with the initial conditions

$$
\begin{aligned}
& u(x, 0)=u^{0}(x), \\
& u_{t}(x, 0)=u^{1}(x), \\
& \theta(x, 0)=\theta^{0}(x)
\end{aligned}
$$

and the boundary conditions

$$
\begin{aligned}
& \left.u\right|_{\partial \Omega}=\left.\frac{\partial u}{\partial v}\right|_{\partial \Omega}=0, \\
& \left.\theta\right|_{\partial \Omega}=0 .
\end{aligned}
$$

To the our best knowledge, the existence of global attractor for thermoelastic coupled beam equations was not considered in the presence of nonlinear structure damping. Here the unknown function $u(x, t)$ is the elevation of the surface of beam; $u^{0}(x)$ and $u^{1}(x)$ are the given initial value functions; the subscript $t$ denotes derivative with respect to $t$ and the assumptions on nonlinear functions $\sigma(\cdot), \phi(\cdot), f_{1}(\cdot), f_{2}(\cdot)$, $g(\cdot)$, and the external force function $q(x)$ will be specified later.

Our fundamental assumptions on $\sigma(\cdot), \phi(\cdot), f_{1}(\cdot), f_{2}(\cdot)$, $g(\cdot)$, and $q(x)$ are given as follows.

Assumption 1. We assume that $\sigma(\cdot) \in C^{1}(R)$ satisfying

$$
\sigma(z) z \geq \widetilde{\sigma}(z) \geq 0, \quad \forall z \geq 0,
$$

where $\widetilde{\sigma}(z)=\int_{0}^{z} \sigma(s) d s$. This condition is promptly satisfied if $\sigma(\cdot)$ is nondecreasing with $\sigma(0)=0$.

Assumption 2. We also assume that $\phi(\cdot) \in C^{1}(R)$ satisfying $\phi(0)=0$ and $\phi(\cdot)$ is nondecreasing and

$$
\phi(s) s \geq 0, \quad \forall s \in R^{+} .
$$

Assumption 3. The function $f_{1}(\cdot): R \rightarrow R$ is of class $C^{1}(R)$ and satisfies $f(0)=0$, and there exist constants $k$ and $\rho \geq 0$ such that

$$
\left|f_{1}(u)-f_{1}(v)\right| \leq k_{1}\left(1+|u|^{\rho}+|v|^{\rho}\right)|u-v|,
$$

$\forall u, v \in R$,

$$
-a_{0} \leq \widehat{f}_{1}(u) \leq \frac{1}{2} f_{1}(u) u+a_{1},
$$

where $\widehat{f}_{1}(z)=\int_{0}^{z} f_{1}(s) d s$.

Assumption 4. The function $f_{2}(\cdot): R \rightarrow R$ is of class $C^{1}(R)$ and satisfies $f_{2}(0)=0$, and there exist constants $k_{2}, k_{3}$ and $\varrho \geq 0$ such that

$$
\begin{array}{r}
\left|f_{2}(\theta)-f_{2}(\widetilde{\theta})\right| \leq k_{2}\left(1+|\theta|^{\varrho}+|\widetilde{\theta}|^{\varrho}\right)|\theta-\widetilde{\theta}|, \\
\forall \theta, \widetilde{\theta} \in R, \\
\left(f_{2}(\theta)-f_{2}(\widetilde{\theta})\right)(\theta-\widetilde{\theta}) \geq k_{3}(\theta-\widetilde{\theta})^{\varrho+2}, \\
\forall \theta, \widetilde{\theta} \in R .
\end{array}
$$

Assumption 5. The function $g(\cdot): R \rightarrow R$ is of class $C^{1}(R)$ and satisfies $g(0)=0$, and there exist constants $k_{4}, k_{5}$ and $r \geq 0$ such that

$$
\begin{aligned}
&(g(u)-g(v))(u-v) \geq k_{4}|u-v|^{r+2}, \quad \forall u, v \in R, \\
&|g(u)-g(v)| \leq k_{5}\left(1+\left|u^{r}\right|+|v|^{r}\right)|u-v|, \\
& \forall u, v \in R .
\end{aligned}
$$

Assumption 6. $q(x) \in L^{2}(\Omega)$.

Under the above assumptions, we prove the existence of global solutions and the existence of a global attractor of extensible beam equation system (8)-(11). And the paper is organized as follows. In Section 2, we introduce some Sobolev spaces. In Section 3, we discuss the existence and uniqueness of global strong solution and weak solution. In Sections 4 and 5, we establish the result of the existence of a global attractor.

\section{Basic Spaces}

Our analysis is based on the following Sobolev spaces. Let

$$
U=\left\{\theta \in H^{2}(\Omega) \cap L^{2}(\Omega) ;\left.\theta\right|_{\partial \Omega}=0\right\} .
$$

Then for regular solutions we consider the phase space

$$
H_{1}=H^{4}(\Omega) \cap H_{0}^{2}(\Omega) \times H^{4}(\Omega) \cap H_{0}^{2}(\Omega) \times U .
$$

In the case of weak solutions we consider the phase space

$$
H_{0}=H_{0}^{2}(\Omega) \times L^{2}(\Omega) \times L^{2}(\Omega) .
$$

In $H_{0}$ we adopt the norm defined by

$$
\|(u, v, \theta)\|_{H_{0}}^{2}=\|\Delta u\|^{2}+\|v\|^{2}+\|\theta\|^{2} .
$$




\section{The Existence of Global Solutions}

Firstly, using the classical Galerkin method, we can establish the existence and uniqueness of regular solution to problem (8)-(11). We state it as follows.

Theorem 7. Under assumptions $\left(H_{1}\right)-\left(H_{6}\right)$, for any initial data $\left(u^{0}, u^{1}, \theta^{0}\right) \in H_{1}$, then problem (8)-(11) has a unique regular solution $(u, \theta)$ with

$$
\begin{aligned}
& u \in L^{\infty}\left(R^{+}, H^{4}(\Omega) \cap H_{0}^{2}(\Omega)\right), \\
& u_{t} \in L^{\infty}\left(R^{+}, H^{4}(\Omega) \cap H_{0}^{2}(\Omega)\right), \\
& u_{t t} \in L^{\infty}\left(R^{+}, L^{2}(\Omega)\right), \\
& \theta \in L^{\infty}\left(R^{+}, U\right), \\
& \theta_{t} \in L^{\infty}\left(R^{+}, L^{2}(\Omega)\right) .
\end{aligned}
$$

Proof. Let us consider the variational problem associated with (8)-(11): find $(u(t), \theta(t)) \in H^{4}(\Omega) \cap H_{0}^{2}(\Omega) \times U$ such that

$$
\begin{aligned}
& \int_{\Omega} u_{t t} \omega d x+\int_{\Omega} \Delta u \Delta \omega d x+\int_{\Omega} \Delta u_{t} \Delta \omega d x \\
& +\sigma\left(\|\nabla u\|^{2}\right) \int_{\Omega} \nabla u \nabla \omega d x \\
& +\phi\left(\int_{\Omega} \nabla u_{t} \nabla \omega d x\right) \int_{\Omega} \nabla u_{t} \nabla \omega d x \\
& +\int_{\Omega} f_{1}(u) \omega d x+\int_{\Omega} g\left(u_{t}\right) \omega d x \\
& +v \int_{\Omega} \Delta \theta \omega d x=\int_{\Omega} q(x) \omega d x \\
& \int_{\Omega} \theta_{t} @ d x-\int_{\Omega} \Delta \theta \omega d x+\int_{\Omega} f_{2}(\theta) \omega d x \\
& \quad-v \int_{\Omega} \Delta u_{t} \boxminus d x=0
\end{aligned}
$$

for all $\omega \in H_{0}^{2}(\Omega)$ and $\omega \in U$. This is done with the Galerkin approximation method which is standard. Here we denote the approximate solution by $\left(u^{m}(t), \theta^{m}(t)\right)$. We can get the theorem by proving the existence of approximation solution, the estimate of approximation solution, convergence, and uniqueness. In the following we give the estimates of approximation solution and the proof of uniqueness of solution.

Estimate 1. In the first approximate equation and the second approximate equation of (25), respectively putting $\omega=u_{t}^{m}(t)$ and $\omega=\theta^{m}(t)$ and making a computation of addition and considering $\widehat{\sigma}(z)=\int_{0}^{z} \sigma(s) d s$ and $\widehat{f}_{1}(z)=\int_{0}^{z} f_{1}(s) d s$, by using Schwarz inequality, and then integrating from 0 to $t<$ $t_{m}$, we see that

$$
\begin{aligned}
\left\|u_{t}^{m}\right\|^{2} & +\left\|\Delta u^{m}\right\|^{2}+\widehat{\sigma}\left(\left\|\nabla u^{m}\right\|^{2}\right)+\int_{\Omega} \widehat{f}_{1}\left(u^{m}\right) d x \\
& +\left\|\theta^{m}\right\|^{2}+\int_{0}^{t}\left\|\Delta u_{t}^{m}\right\|^{2} d t+2 \int_{0}^{t}\left\|\nabla \theta^{m}\right\|^{2} d t \\
& +2 \int_{0}^{t} \phi\left(\int_{\Omega} \nabla u \nabla u_{t} d x\right) \int_{\Omega} \nabla u \nabla u_{t} d x d s \\
& +2 \int_{0}^{t} \int_{\Omega} g\left(u_{t}^{m}\right) u_{t}^{m} d x d s \\
& +2 \int_{0}^{t} \int_{\Omega} f_{2}\left(\theta^{m}\right) \theta^{m} d x d s \\
\leq & \frac{1}{\lambda_{1}} \int_{0}^{t}\|q(x)\|^{2} d s+\left\|u_{t}^{m}(0)\right\|^{2}+\left\|\Delta u^{m}(0)\right\|^{2} \\
& +\widehat{\sigma}\left(\left\|\nabla u^{m}(0)\right\|^{2}\right)+\int_{\Omega} \widehat{f}_{1}\left(u^{m}(0)\right) d x \\
& +\left\|\theta^{m}(0)\right\|^{2}
\end{aligned}
$$

independent of $m$ and $t$, where $\lambda_{1}$ is the same as $\lambda_{1}$ of (47). Taking into account assumptions (13), (17), and (18) of $\phi(\cdot)$, $f_{2}(\cdot)$, and $g(\cdot)$ and Assumption 6, we see that there exists $M_{1}>0$ depending only on $T$ such that

$$
\begin{gathered}
\left\|u_{t}^{m}\right\|^{2}+\left\|\Delta u^{m}\right\|^{2}+\widehat{\sigma}\left(\left\|\nabla u^{m}\right\|^{2}\right)+\int_{\Omega} \widehat{f}_{1}\left(u^{m}\right) d x \\
+\left\|\theta^{m}\right\|^{2} \leq M_{1}
\end{gathered}
$$

for all $t \in[0, T]$ and for all $m \in N$.

Estimate 2. In the first approximate equation and the second approximate equation of (25), respectively, putting $\omega=$ $\Delta^{2} u_{t}^{m}(t)$ and $\omega=\Delta \theta^{m}(t)$ and making a computation of addition by using Schwarz inequality and Young inequality and considering the assumptions of $\sigma(\cdot), \phi(\cdot), f_{1}(\cdot), g(\cdot), f_{2}(\cdot)$, and $q(\cdot)$, we see that there exists $M_{2}>0$ depending only on $T$ such that

$$
\left\|\Delta u_{t}^{m}\right\|^{2}+\left\|\Delta^{2} u^{m}\right\|^{2}+\left\|\nabla \theta^{m}\right\|^{2} \leq M_{2}
$$

for all $t \in[0, T]$ and for all $m \in N$.

Estimate 3. In the first approximate equation and the second approximate equation of (25), respectively integrating by parts with $\omega=u_{t t}^{m}(0)$ and $\omega=\theta_{t}^{m}(0)$ with $t=0$ and using Schwarz inequality and Young inequality, we see that there exists $M_{3}, M_{4}>0$ depending only on $T$ such that

$$
\begin{gathered}
\left\|u_{t t}^{m}(0)\right\|^{2} \leq M_{3}, \\
\left\|\theta_{t}^{m}(0)\right\|^{2} \leq M_{4}
\end{gathered}
$$

for all $t \in[0, T]$ and for all $m \in N$. 
Estimate 4. Let us fix $t, \xi>0$ such that $\xi<T-t$. Respectively taking the difference of the first approximate equation and the second approximate equation of (25) with $t=t+\xi$ and $t=t$ and respectively replacing $\omega$ by $u_{t}^{m}(t+\xi)-u_{t}^{m}(t)$ and $\omega$ by $\theta^{m}(t+\xi)-\theta^{m}(t)$, we can find constants $M_{5}, M_{6}>0$, depending only on $T$, such that

$$
\begin{aligned}
\left\|u_{t t}^{m}\right\|^{2}+\left\|\Delta u_{t}^{m}\right\|^{2} & \leq M_{5}, \\
\left\|\theta_{t}^{m}\right\|^{2} & \leq M_{6},
\end{aligned}
$$

$\forall m \in N, \forall t \in[0, T]$.

Estimate 5. Taking the scalar product in $H$ with $\omega=\Delta \theta^{m}$ for the second approximate equation of (25), after a computation we can find a constant $M_{7}>0$, depending only on $T$ such that

$$
\left\|\Delta \theta^{m}\right\|^{2} \leq M_{7}, \quad \forall m \in N, \forall t \in[0, T] .
$$

With the estimates 1-2 and 4-5, we can get the necessary compactness in order to pass approximate equation of (25) to the limit. Then it is a matter of routine to conclude the existence of global solutions in $[0, T]$.

Uniqueness. Let $(u, \theta),(v, \widetilde{\theta})$ be two solutions of (8)-(11) with the same initial data. Then writing $p=u-v, \vartheta=\theta-\widetilde{\theta}$ and taking the difference (25) with $u=u, \theta=\theta$ and $u=v, \theta=\widetilde{\theta}$ and respectively replacing $\omega, \omega$ by $p_{t}, \vartheta$ and then making a computation of addition, we have

$$
\begin{aligned}
\frac{1}{2} \frac{d}{d t}[ & {\left[\left\|p_{t}\right\|^{2}+\|\Delta p\|^{2}+\|\vartheta\|^{2}\right]+\left\|\Delta p_{t}\right\|^{2}+\|\Delta \vartheta\|^{2}+J_{1} } \\
& +J_{2}+\int_{\Omega}\left[f_{1}(u)-f_{1}(v)\right] p_{t} d x \\
& +\int_{\Omega}\left[g\left(u_{t}\right)-g\left(v_{t}\right)\right] p_{t} d x \\
& +\int_{\Omega}\left[f_{2}(\theta)-f_{2}(\widetilde{\theta})\right] \vartheta_{t} d x=0,
\end{aligned}
$$

where $J_{1}=\int_{\Omega}\left[\sigma\left(\int_{\Omega}(\nabla u)^{2} d x\right) \Delta u-\sigma\left(\int_{\Omega}(\nabla v)^{2} d x\right) \Delta v\right] p_{t} d x$ and $J_{2}=\int_{\Omega}\left[\phi\left(\int_{\Omega} \nabla u \nabla u_{t} d x\right) \Delta u-\phi\left(\int_{\Omega} \nabla v \nabla v_{t} d x\right) \Delta v\right] p_{t} d x$. Using Mean Value Theorem and the Young inequalities combined with the estimates 1-2 and 4-5, we deduce that for some constant $M_{8}>0$,

$$
\begin{aligned}
& \frac{d}{d t}\left(\left\|p_{t}\right\|^{2}+\|\Delta p\|^{2}+\|\vartheta\|^{2}\right) \\
& \quad \leq M_{8}\left(\left\|p_{t}\right\|^{2}+\|\Delta p\|^{2}+\|\vartheta\|^{2}\right), \quad \forall t \in(0, T) .
\end{aligned}
$$

Then from Gronwall's Lemma we see that $u=v, \theta=\widetilde{\theta}$. The proof of Theorem 7 is completed.

Theorem 8. Under the assumptions of Theorem 7 , if the initial data $\left(u^{0}, u^{1}, \theta^{0}\right) \in H_{0}$, there exists a unique weak solution of problem (8)-(11) which depends continuously on initial data with respect to the norm of $H_{0}$.
Proof. By using density arguments, we can obtain the existence of a weak solution in $H_{0}$.

Let us consider $\left\{u^{0}, u^{1}, \theta^{0}\right\} \in \overline{H_{1}}$. Since $H_{1}$ is dense in $H_{0}$, then there exists $\left\{u_{\mu}^{0}, u_{\mu}^{1}, \theta_{\mu}^{0}\right\} \subset H_{1}$, such that

$$
\begin{aligned}
& u_{\mu}^{0} \longrightarrow u^{0} \quad \text { in } H_{0}^{2}(\Omega) ; \\
& u_{\mu}^{1} \longrightarrow u^{1} \quad \text { in } L^{2}(\Omega) ; \\
& \theta_{\mu}^{0} \longrightarrow \theta^{0} \quad \text { in } L^{2}(\Omega) .
\end{aligned}
$$

We observe that for each $\mu \in N$, there exists $\left(u_{\mu}, \theta_{\mu}\right)$, smooth solution of the initial-boundary value problem (8)-(11) which satisfies

$$
\begin{aligned}
& u_{t t \mu}+\Delta^{2} u_{\mu}+\Delta^{2} u_{t \mu} \\
& -\left[\sigma\left(\int_{\Omega}\left(\nabla u_{\mu}\right)^{2} d x\right)+\phi\left(\int_{\Omega} \nabla u_{\mu} \nabla u_{t \mu} d x\right)\right] \Delta u_{\mu} \\
& \quad+f_{1}\left(u_{\mu}\right)+g\left(u_{t \mu}\right)+v \Delta \theta_{\mu}=q(x), \\
& \theta_{t \mu}-\Delta \theta_{\mu}+f_{2}\left(\theta_{\mu}\right)-v \Delta u_{t \mu}=0 .
\end{aligned}
$$

Respectively multiplying the first equation in (35) by $u_{\mu}$ and multiplying the second equation in (35) by $\theta_{\mu}$ and integrating over $\Omega$ and taking the sum and then considering the arguments used in the estimate of the existence of solution, we obtain

$$
\left\|u_{t \mu}\right\|^{2}+\left\|\Delta u_{\mu}\right\|^{2}+\left\|\theta_{\mu}\right\|^{2} \leq C_{0},
$$

where $C_{0}$ is a positive constant independent of $\mu \in N$.

Defining $Z_{\mu, \sigma}=u_{\mu}-u_{\sigma}, \widetilde{Z}_{\mu, \sigma}=\theta_{\mu}-\widetilde{\theta}_{\mu}: \mu, \sigma \in N$, following the steps already used in the uniqueness of regular solution for (8)-(11), and considering the convergence given in (34), we deduce that there exists $(u, \theta)$ such that

$$
\begin{gathered}
u_{\mu} \longrightarrow u \quad \text { strongly in } C\left([0, T) ; H_{0}^{2}(\Omega)\right), \\
u_{t \mu} \longrightarrow u_{t} \quad \text { strongly in } C\left([0, T) ; L^{2}(\Omega)\right), \\
\theta_{\mu} \longrightarrow \theta \quad \text { strongly in } C\left([0, T) ; L^{2}(\Omega)\right) .
\end{gathered}
$$

From the above convergence, we can pass to the limit using standard arguments in order to obtain

$$
\begin{aligned}
& u_{t t \mu}+\Delta^{2} u_{\mu}+\Delta^{2} u_{t \mu} \\
& -\left[\sigma\left(\int_{\Omega}\left(\nabla u_{\mu}\right)^{2} d x\right)+\phi\left(\int_{\Omega} \nabla u_{\mu} \nabla u_{t \mu} d x\right)\right] \Delta u_{\mu} \\
& \quad+f_{1}\left(u_{\mu}\right)+g\left(u_{t \mu}\right)+v \Delta \theta_{\mu}=q(x), \\
& \theta_{t \mu}-\Delta \theta_{\mu}+f_{2}\left(\theta_{\mu}\right)-v \Delta u_{t \mu}=0 .
\end{aligned}
$$

Theorem 8 is proved.

Remark 9. In both cases

$$
\left\|u_{t}\right\|^{2}+\|\Delta u\|^{2}+\|u\|_{\rho+2}^{\rho+2}+\|\theta\|^{2} \leq C,
$$

where $C$ is a constant depending on the initial data in different expression. 
In addition, in this paper, $C$ denotes different constant in different expression.

Remark 10. Theorem 8 implies that problem (8)-(11) defines a nonlinear $C_{0}$-semigroup $S(t)$ on $H_{0}$. Indeed, let us set $S(t)\left(u^{0}, u^{1}, \theta^{0}\right)=\left(u(t), u_{t}(t), \theta(t)\right)$, where $u$ is the unique solution corresponding to initial data $\left(u^{0}, u^{1}, \theta^{0}\right) \in H_{0}$. Moreover, the operator $S(t)$ defined in $H_{0}$ meets the usual semigroup properties

$$
\begin{aligned}
S(t, \tau) & =S(t) S(\tau), \quad \forall t, \tau \in R, \\
S(0) & =I .
\end{aligned}
$$

To prove the main result, we need the following Lemma 11 of Nakao and Lemma 12

Lemma 11 (see [16]). Let $\varphi(t)$ be a nonnegative continuous function defined on $[0, T), 1<T \leq \infty$, which satisfies

$$
\begin{aligned}
\sup _{t \leq s \leq t+1} \varphi(s)^{1+\eta} \leq M_{0}(\varphi(t)-\varphi(t+1))+ & M_{1}, \\
& 0 \leq t \leq T-1,
\end{aligned}
$$

where $M_{0}, M_{1}$, $\eta$ are positive constants. Then we have

$$
\begin{aligned}
\varphi(t) \leq & \left(M_{0}^{-1} \eta(t-1)^{+}+\left(\sup _{0 \leq s \leq 1} \varphi(s)\right)^{-\eta}\right)^{-1 / \eta} \\
& +M_{1}^{1 /(\eta+1)}, \quad 0 \leq t \leq T
\end{aligned}
$$

Lemma 12 (see [17]). Assume that for any bounded positive invariant set $B \subset H$ and for any $\varepsilon>0$, there exists $T=T(\varepsilon, B)$ such that

$$
d(S(T) x, S(T) y) \leq \varepsilon+\omega_{T}(x, y), \quad \forall x, y \in B,
$$

where $\omega_{T}: H \times H \rightarrow R$ satisfies for any sequence $\left\{z_{n}\right\} \subset B$

$$
\liminf _{m \rightarrow \infty} \liminf _{n \rightarrow \infty} \omega_{T}\left(z_{n}, z_{m}\right)=0 .
$$

Then $S(t)$ is asymptotically smooth.

\section{The Existence of Absorbing Set}

The main result of an absorbing set reads as follows.

Theorem 13. Assume the hypotheses of Theorem 8; then the corresponding semigroup $S(t)$ of problem (8)-(11) has an absorbing set $\mathbb{B}$ in $\mathrm{H}_{0}$.

Proof. Now we show that semigroup $S(t)$ has as absorbing set $\mathbb{B}$ in $H_{0}$. Firstly, we can calculate the total energy functional

$$
\begin{aligned}
E(t)= & \frac{1}{2}\left\{\left\|u_{t}\right\|^{2}+\|\Delta u\|^{2}+\widehat{\sigma}\left(\|\nabla u\|^{2}\right)+\|\theta\|^{2}\right\} \\
& +\int_{\Omega} \widehat{f}_{1}(u) d x-\int_{\Omega} q u(t) d x .
\end{aligned}
$$

Let us fix an arbitrary bounded set $B \subset H_{0}$ and consider the solutions of problem (8)-(11) given by $\left(u(t), u_{t}(t), \theta(t)\right)=$
$S(t)\left(u^{0}, u^{1}, \theta^{0}\right)$ with $\left(u^{0}, u^{1}, \theta^{0}\right) \in B$. Our analysis is based on the modified energy function

$$
\widetilde{E}(t)=E(t)+a_{0}|\Omega|+\frac{1}{\lambda_{1}}\|q\|^{2},
$$

where $\lambda_{1}>0$ is the first eigenvalue of the operator $\Delta$ in $H_{0}^{2}(\Omega)$; that is, $\lambda_{1}$ satisfies

$$
\|u\|^{2} \leq \frac{1}{\lambda_{1}}\|\Delta u\|^{2}, \quad \forall u \in H_{0}^{2}(\Omega) .
$$

It is easy to see that $\widetilde{E}(t)$ dominates $\left\|\left(u(t), u_{t}(t), \theta(t)\right)\right\|_{H_{0}}^{2}$ and $\widetilde{E}(t) \geq(1 / 4)\|\Delta u(t)\|^{2}$. By multiplying (8) by $u$ and integrating over $\Omega$, we have

$$
\begin{aligned}
\|\Delta u\|^{2} & =-\left[\sigma\left(\int_{\Omega}|\nabla u|^{2} d x\right)+\phi\left(\int_{\Omega} \nabla u \nabla u_{t}\right) d x\right]\|\nabla u\|^{2} \\
& -\int_{\Omega} f_{1}(u) u d x+\left\|u_{t}\right\|^{2}-\frac{d}{d t}\left(u_{t}, u\right) \\
& -\int_{\Omega} g\left(u_{t}\right) u d x+\int_{\Omega} q u(t) d x-\int_{\Omega} \Delta u \Delta u_{t} d x \\
& +v \int_{\Omega} \nabla \theta \nabla u d x .
\end{aligned}
$$

Inserting (48) into $\widetilde{E}(t)$, we obtain

$$
\begin{aligned}
\widetilde{E}(t) & \left\|u_{t}\right\|^{2} \\
& +\frac{1}{2}\left[\widehat{\sigma}\left(\int_{\Omega}|\nabla u|^{2} d x\right)-\sigma\left(\int_{\Omega}|\nabla u|^{2} d x\right)\|\nabla u\|^{2}\right] \\
& -\frac{1}{2} \phi\left(\int_{\Omega} \nabla u \nabla u_{t} d x\right)\|\nabla u\|^{2}+\frac{1}{2}\|\theta\|^{2} \\
& -\frac{1}{2} \int_{\Omega} \Delta u \Delta u_{t} d x+\int_{\Omega}\left(\widehat{f}_{1}(u)-\frac{1}{2} f_{1}(u) u\right) d x \\
& -\frac{1}{2} \frac{d}{d t} \int_{\Omega} u_{t} u d x-\frac{1}{2} \int_{\Omega} g\left(u_{t}\right) u d x \\
& +\frac{v}{2} \int_{\Omega} \nabla \theta \nabla u d x+a_{0}|\Omega|+\frac{1}{\lambda_{1}}\|q\|^{2} \\
& -\frac{1}{2} \int_{\Omega} q u(t) d x .
\end{aligned}
$$

Considering (12) and (15) and integrating from $t_{1}$ to $t_{2}$ for (49), we obtain that

$$
\begin{aligned}
& \int_{t_{1}}^{t_{2}} \widetilde{E}(t) d s \\
& \quad \leq \int_{t_{1}}^{t_{2}}\left\|u_{t}\right\|^{2} d s-\frac{1}{2} \int_{t_{1}}^{t_{2}} \phi\left(\int_{\Omega} \nabla u \nabla u_{t} d x\right)\|\nabla u\|^{2} d s
\end{aligned}
$$




$$
\begin{aligned}
& +\frac{1}{2} \int_{t_{1}}^{t_{2}}\|\theta\|^{2} d s-\frac{1}{2} \int_{t_{1}}^{t_{2}} \int_{\Omega} \Delta u \Delta u_{t} d x d s \\
& -\frac{1}{2}\left(\int_{\Omega} u_{t}\left(t_{2}\right) u\left(t_{2}\right) d x-\int_{\Omega} u_{t}\left(t_{1}\right) u\left(t_{1}\right) d x\right) \\
& -\frac{1}{2} \int_{t_{1}}^{t_{2}} \int_{\Omega} g\left(u_{t}\right) u d x d s \\
& +\frac{v}{2} \int_{t_{1}}^{t_{2}} \int_{\Omega} \nabla \theta \nabla u d s d x+\left(a_{0}+a_{1}\right)|\Omega| \\
& +\frac{1}{\lambda_{1}}\|q\|^{2}+\frac{1}{2} \int_{t_{1}}^{t_{2}} \int_{\Omega} q u(t) d x d s,
\end{aligned}
$$

where $t_{1}, t_{2} \in[t, t+1]$.

Now let us begin to estimate the right hand side of (50) to use the above Lemma 11 of Nakao.

First, by multiplying (8) by $u_{t}$ and multiplying (9) by $\theta$ and integrating over $\Omega$ and then taking the sum, we have

$$
\begin{aligned}
\frac{d}{d t} E(t) & +\left\|\Delta u_{t}\right\|^{2}+\|\nabla \theta\|^{2} \\
= & -\int_{\Omega} g\left(u_{t}\right) u_{t} d x \\
& -\phi\left(\int_{\Omega} \nabla u \nabla u_{t} d x\right) \int_{\Omega} \nabla u \nabla u_{t} d x \\
& -\int_{\Omega} f_{2}(\theta) \theta d x .
\end{aligned}
$$

Then integrating from $t$ to $t+1$, we get

$$
\begin{aligned}
E(t+1)-E(t)+\int_{t}^{t+1}\left\|\Delta u_{t}\right\|^{2} d s+\int_{t}^{t+1}\|\nabla \theta\|^{2} d s \\
=-\int_{t}^{t+1} \int_{\Omega} g\left(u_{t}\right) u_{t} d x d s \\
\quad-\int_{t}^{t+1} \phi\left(\int_{\Omega} \nabla u \nabla u_{t} d x\right) \int_{\Omega} \nabla u \nabla u_{t} d x d s \\
\quad-\int_{t}^{t+1} \int_{\Omega} f_{2}(\theta) \theta d x d s .
\end{aligned}
$$

Taking into account assumptions (13), (17), and (18) of $\phi(\cdot)$, $f_{2}(\cdot)$, and $g(\cdot)$, we have

$$
E(t) \geq E(t+1) .
$$

Then we define an auxiliary function $I^{2}(t)$ by putting

$$
I^{2}(t)=E(t)-E(t+1) \geq 0 .
$$

Thus it is obvious that

$$
\begin{gathered}
\int_{t}^{t+1}\left\|\Delta u_{t}\right\|^{2} \leq I^{2}(t), \\
\int_{t}^{t+1}\|\nabla \theta\|^{2} \leq I^{2}(t) .
\end{gathered}
$$

Noting that $r /(r+2)+2 /(r+2)=1$ and using twice Holder inequalities and considering assumption (18) of $g(\cdot)$, we have

$$
\begin{aligned}
& \int_{t}^{t+1}\left\|u_{t}\right\|^{2} d s \\
& \quad \leq|\Omega|^{r /(r+2)}\left(\int_{t}^{t+1} \int_{\Omega}\left|u_{t}\right|^{(r+2)} d x d s\right)^{2 /(r+2)} \\
& \quad \leq|\Omega|^{r /(r+2)} \frac{1}{k_{4}}\left(\int_{t}^{t+1} \int_{\Omega} g\left(u_{t}\right) u_{t} d x d s\right)^{2 /(r+2)} \\
& \quad \leq|\Omega|^{r /(r+2)} \frac{1}{k_{4}} I(t)^{4 /(r+2)} .
\end{aligned}
$$

Using the Mean Value Theorem with $\phi(0)=0$ and considering the estimate of (39) and then using Young inequality combined with (55), we have

$$
\begin{aligned}
& \frac{1}{2} \int_{t_{1}}^{t_{2}} \phi\left(\int_{\Omega} \nabla u \nabla u_{t} d x\right)\|\nabla u\|^{2} d s \\
& \quad=\frac{1}{2} \int_{t_{1}}^{t_{2}} \phi^{\prime}\left(\xi_{1}\right) \int_{\Omega} \nabla u \nabla u_{t} d x\|\nabla u\|^{2} d s \\
& \quad \leq \frac{1}{2} \int_{t_{1}}^{t_{2}} C\left\|\Delta u_{t}\right\|\|\Delta u\| d s \\
& \quad \leq \frac{C}{4 \eta} \int_{t_{1}}^{t_{2}}\left\|\Delta u_{t}\right\|^{2} d s+\frac{\eta}{4} \int_{t_{1}}^{t_{2}}\|\Delta u\|^{2} d s \\
& \quad \leq \frac{C}{4 \eta} \int_{t_{1}}^{t_{2}}\left\|\Delta u_{t}\right\|^{2} d s+\eta \sup _{t \leq s \leq t+1} \widetilde{E}(s) \\
& \quad \leq \frac{C}{4 \eta} I^{2}(t)+\eta \sup _{t \leq s \leq t+1} \widetilde{E}(s),
\end{aligned}
$$

where $\xi_{1}$ is among 0 and $\int_{\Omega} \nabla u \nabla u_{t} d x$.

Since $\|\theta\|^{2} \leq\left(1 / \lambda_{2}\right)\|\nabla \theta\|^{2}, \forall \theta \in H_{0}^{1}$, from (56) we obtain

$$
\frac{1}{2} \int_{t_{1}}^{t_{2}}\|\theta\|^{2} d s \leq \frac{1}{2 \lambda_{2}} I^{2}(t),
$$

where $\lambda_{2}$ is the first eigenvalue of the operator $\nabla$ in $H_{0}^{1}(\Omega)$.

Using Young inequality, we get

$$
\begin{aligned}
\frac{1}{2} \int_{t_{1}}^{t_{2}} \int_{\Omega} \Delta u \Delta u_{t} d s \leq & \frac{1}{4 \eta} \int_{t_{1}}^{t_{2}}\left\|\Delta u_{t}\right\|^{2} d s \\
& +\frac{\eta}{4} \int_{t_{1}}^{t_{2}}\|\Delta u\|^{2} d s \\
\leq & \frac{1}{4 \eta} I(t)^{2}+\eta \sup _{t \leq s \leq t+1} \widetilde{E}(s) .
\end{aligned}
$$


Since (57), in view of the Mean Value Theorem for integral, there exist number $t_{1} \in[t, t+1 / 4]$ and number $t_{2} \in[t+$ $3 / 4, t+1]$ such that

$$
\begin{aligned}
\left\|u_{t}\left(t_{1}\right)\right\|^{2} & \leq|\Omega|^{r /(r+2)} \frac{4}{k_{4}} I(t)^{4 /(r+2)}, \\
\left\|u_{t}\left(t_{2}\right)\right\|^{2} & \leq|\Omega|^{r /(r+2)} \frac{4}{k_{4}} I(t)^{4 /(r+2)} .
\end{aligned}
$$

Thus from Schwarz inequality combined with (47) and (61), we have

$$
\begin{aligned}
& \frac{1}{2}\left(\int_{\Omega} u_{t}\left(t_{2}\right) u\left(t_{2}\right) d x-\int_{\Omega} u_{t}\left(t_{1}\right) u\left(t_{1}\right) d x\right) \\
& \quad \leq \frac{1}{2 \sqrt{\lambda_{1}}}\left(\left\|u_{t}\left(t_{2}\right)\right\|\left\|\Delta u\left(t_{2}\right)\right\|+\left\|u_{t}\left(t_{1}\right)\right\|\left\|\Delta u\left(t_{1}\right)\right\|\right) \\
& \quad \leq \frac{2}{\sqrt{\lambda_{1}} \sqrt{k_{4}}}|\Omega|^{r / 2(r+2)} I(t)^{2 /(r+2)} \sup _{t \leq s \leq t+1}\|\Delta u\| \\
& \quad \leq \frac{\left(\left(2 / \sqrt{\lambda_{1}} \sqrt{k_{4}}\right)|\Omega|^{r / 2(r+2)}\right)^{2}}{\eta} I(t)^{4 /(r+2)} \\
& \quad+\eta \sup _{t \leq s \leq t+1} \widetilde{E}(s) .
\end{aligned}
$$

Considering assumption (19) of $g(\cdot)$ and using Young inequality and Holder inequality with $(r+1) /(r+2)+1 /(r+$ $2)=1$, then from (47) and (57), we have

$$
\begin{aligned}
& \frac{1}{2} \int_{t_{1}}^{t_{2}} \int_{\Omega} g\left(u_{t}\right) u d x d s \leq \frac{k_{5}}{2} \int_{t_{1}}^{t_{2}} \int_{\Omega}\left(1+\left|u_{t}\right|^{r}\right)\left|u_{t}\right| u d x d s \\
& \leq \frac{k_{5}^{2}}{4 \eta \lambda_{1}} \int_{t}^{t+1} \int_{\Omega} u_{t}(s)^{2} d x d s+\frac{\eta \lambda_{1}}{4} \int_{t}^{t+1} \int_{\Omega} u(s)^{2} d x d s \\
& +\frac{k_{5}}{2} \int_{t_{1}}^{t_{2}}\left|u_{t}\right|^{r+1} u d x d s \leq \frac{k_{5}^{2}}{4 \eta \lambda_{1}} \int_{t}^{t+1} \int_{\Omega} u_{t}(s)^{2} d x d s+\frac{\eta \lambda_{1}}{4} \\
& \cdot \int_{t}^{t+1} \int_{\Omega} u(s)^{2} d x d s+\frac{k_{5}}{2} \\
& . \int_{t}^{t+1}\left(\int_{\Omega}\left|u_{t}\right|^{r+2} d x\right)^{(r+1) /(r+2)}\left(\int_{\Omega} u^{r+2} d x\right)^{1 /(r+2)} d s \\
& \quad \leq \frac{k_{5}^{2}}{4 \eta \lambda_{1}} \int_{t}^{t+1} \int_{\Omega} u_{t}(s)^{2} d x d s+\frac{\eta \lambda_{1}}{4} \int_{t}^{t+1} \int_{\Omega} u(s)^{2} d x d s \\
& \quad+\frac{k_{5}}{2}\left\{\int_{t}^{t+1}\left[\left(\int_{\Omega} u_{t}^{r+2} d x\right)^{(r+1) /(r+2)}\right]^{(r+2) /(r+1)} d s\right\}^{(r+1) /(r+2)} \\
& \quad \cdot\left\{\int_{t}^{t+1}\left[\left(\int_{\Omega} u^{r+2} d x\right)^{1 /(r+2)}\right]^{r+2} d s\right\}^{1 /(r+2)}=\frac{k_{5}^{2}}{4 \eta \lambda_{1}} \\
& \quad+\frac{k_{5}}{2}\left(\int_{t}^{t+1} \int_{\Omega}^{t+1} \int_{\Omega} u_{t}^{r+2} d x d s\right)^{(r+1) /(r+2)} d x d s+\frac{\eta \lambda_{1}}{4} \int_{t}^{t+1} \int_{\Omega} u(s)^{2} d x d s
\end{aligned}
$$

$$
\begin{aligned}
& \cdot\left(\int_{t}^{t+1} \int_{\Omega} u^{r+2} d x d s\right)^{1 /(r+2)} \leq \frac{k_{5}^{2}}{4 \eta \lambda_{1}}|\Omega|^{r /(r+2)} \frac{1}{k_{4}} I(t)^{4 /(r+2)} \\
& +\eta \sup _{t \leq s \leq t+1} \widetilde{E}(s)+\frac{k_{5} \mu}{2 k_{4}} I(t)^{2(r+1) /(r+2)} \sup _{t \leq s \leq t+1}\|\Delta u\| \\
& \leq \frac{k_{5}^{2}}{4 \eta \lambda_{1}}|\Omega|^{r /(r+2)} \frac{1}{k_{4}} I(t)^{4 /(r+2)}+\eta \sup _{t \leq s \leq t+1} \widetilde{E}(s)+\frac{k_{5}^{2} \mu^{2}}{4 \eta k_{4}^{2}} \\
& \cdot I(t)^{4(r+1) /(r+2)}+\eta \sup _{t \leq s \leq t+1} \widetilde{E}(s) .
\end{aligned}
$$

Also by Young inequality, we have

$$
\begin{aligned}
\frac{\nu}{2} \int_{t_{1}}^{t_{2}} \int_{\Omega} \nabla \theta \nabla u d x d s \leq & \frac{\nu}{2} \int_{t_{1}}^{t_{2}}\|\nabla \theta\|\|\nabla u\| d s \\
& \leq \int_{t_{1}}^{t_{2}} \frac{v^{2}}{4 \eta \lambda_{3}}\|\nabla \theta\|^{2} d s \\
& +\int_{t_{1}}^{t_{2}} \frac{\eta \lambda_{3}}{4}\|\nabla u\|^{2} d s \\
& \leq \frac{v^{2}}{4 \eta \lambda_{3}} I^{2}(t)+\eta \sup _{t \leq s \leq t+1} \widetilde{E}(s),
\end{aligned}
$$

where $\lambda_{3}>0$ is the first eigenvalue of the operator $\nabla$ in $H_{0}^{1}(\Omega)$; that is, $\lambda_{3}$ satisfies

$$
\|\nabla u\|^{2} \leq \frac{1}{\lambda_{3}}\|\Delta u\|^{2}, \quad \forall u \in H_{0}^{1}(\Omega) .
$$

Finally using Young inequality again, we get that

$$
\frac{1}{2} \int_{t_{1}}^{t_{2}} \int_{\Omega} q u(t) d x d s \leq \frac{1}{4 \eta \lambda_{1}}\|q\|^{2}+\eta \sup _{t \leq s \leq t+1} \widetilde{E}(s) .
$$

Inserting (57)-(60) and (62), (63), (64), and (66) into (50), we obtain

$$
\begin{aligned}
& \int_{t_{1}}^{t_{2}} \widetilde{E}(s) d s \leq\left[\left(|\Omega|^{r /(r+2)} \frac{1}{k_{4}}\right.\right. \\
& +\frac{\left(\left(2 / \sqrt{\lambda_{1}} \sqrt{k_{4}}\right)|\Omega|^{r / 2(r+2)}\right)^{2}}{\eta} \\
& \left.+\frac{k_{5}^{2}}{4 \eta \lambda_{1}}|\Omega|^{r /(r+2)} \frac{1}{k_{4}}\right) I(t)^{4 /(r+2)}+\left(\frac{C}{4 \eta}+\frac{1}{2 \lambda_{2}}\right. \\
& \left.\left.+\frac{1}{4 \eta}+\frac{\nu^{2}}{4 \eta \lambda_{3}}\right) I(t)^{2}+\left(\frac{k_{5}^{2} \mu^{2}}{4 \eta k_{4}^{2}}\right) I(t)^{4(r+1) /(r+2)}\right] \\
& +6 \eta \sup _{t \leq s \leq t+1} \widetilde{E}(s)+\left(a_{0}+a_{1}\right)|\Omega|+\left(\frac{1}{\lambda_{1}}+\frac{1}{4 \eta \lambda_{1}}\right) \\
& +\|q\|^{2} .
\end{aligned}
$$


For the left hand side of (67), we use the Mean Value Theorem; then there exists number $\tau \in\left[t_{1}, t_{2}\right]$ such that

$$
\int_{t_{1}}^{t_{2}} \widetilde{E}(s) d s \geq \frac{1}{2} \widetilde{E}(t+1)=\frac{1}{2}\left(\widetilde{E}(t)-I(t)^{2}\right) .
$$

So we conclude that

$$
\widetilde{E}(t) \leq I(t)^{2}+2 \int_{t_{1}}^{t_{2}} \widetilde{E}(s) d s
$$

Inserting (67) into (69), we obtain that

$$
\begin{aligned}
& \widetilde{E}(t) \leq 2\left[\left(|\Omega|^{r /(r+2)} \frac{1}{k_{4}}\right.\right. \\
& +\frac{\left(\left(2 / \sqrt{\lambda_{1}} \sqrt{k_{4}}\right)|\Omega|^{r / 2(r+2)}\right)^{2}}{\eta} \\
& \left.+\frac{k_{5}^{2}}{8 \eta \lambda_{1}}|\Omega|^{r /(r+2)} \frac{1}{k_{4}}\right) I(t)^{4 /(r+2)}+\left(1+\frac{C}{4 \eta}\right. \\
& \left.+\frac{1}{2 \lambda_{2}}+\frac{1}{4 \eta}+\frac{\nu^{2}}{4 \eta \lambda_{3}}\right) I(t)^{2}+\left(\frac{k_{5}^{2} \mu^{2}}{4 \eta k_{4}^{2}}\right) \\
& +I(t)^{4(r+1) /(r+2)}+12 \eta \sup _{t \leq s \leq t+1} \widetilde{E}(s)+\left(a_{0}+a_{1}\right)|\Omega| \\
& +2\left(\frac{1}{\lambda_{1}}+\frac{1}{4 \eta \lambda_{1}}\right)\|q\|^{2} .
\end{aligned}
$$

Letting $0<\eta<1 / 12$ and noting that $I(t)^{2 r /(r+2)}$ and $I(t)^{4 r /(r+2)}$ are bounded with estimate (39), then from (70), we get

$$
\begin{aligned}
& \widetilde{E}(t) \leq \frac{2}{1-12 \eta} I(t)^{4 /(r+2)}\left[\left(|\Omega|^{r /(r+2)} \frac{1}{k_{4}}\right.\right. \\
& +\frac{\left(\left(2 / \sqrt{\lambda_{1}} \sqrt{k_{4}}\right)|\Omega|^{r / 2(r+2)}\right)^{2}}{\eta} \\
& \left.+\frac{k_{5}^{2}}{4 \eta \lambda_{1}}|\Omega|^{r /(r+2)} \frac{1}{k_{4}}\right)+\left(1+\frac{C}{4 \eta}+\frac{1}{2 \lambda_{2}}+\frac{1}{4 \eta}\right. \\
& \left.\left.+\frac{\nu^{2}}{4 \eta \lambda_{3}}\right) C+\frac{k_{5}^{2} \mu^{2}}{4 \eta k_{4}^{2}} C\right]+\frac{1}{1-12 \eta}\left(a_{0}+a_{1}\right)|\Omega| \\
& +\frac{2}{1-12 \eta}\left(\frac{1}{\lambda_{1}}+\frac{1}{4 \eta \lambda_{1}}\right)\|q\|^{2},
\end{aligned}
$$

where $C$ is a constant which depends on $B$.
Set

$$
\begin{aligned}
C_{1} & =\frac{2}{1-12 \eta}\left[\left(|\Omega|^{r /(r+2)} \frac{1}{k_{4}}\right.\right. \\
& +\frac{\left(\left(2 / \sqrt{\lambda_{1}} \sqrt{k_{4}}\right)|\Omega|^{r / 2(r+2)}\right)^{2}}{\eta} \\
& \left.+\frac{k_{5}^{2}}{4 \eta \lambda_{1}}|\Omega|^{r /(r+2)} \frac{1}{k_{4}}\right)+\left(1+\frac{C}{4 \eta}+\frac{1}{2 \lambda_{2}}+\frac{1}{4 \eta}\right. \\
& \left.\left.+\frac{v^{2}}{4 \eta \lambda_{3}}\right) C+\frac{k_{5}^{2} \mu^{2}}{4 \eta k_{4}^{2}} C\right] ;
\end{aligned}
$$

then (70) can be rewritten as

$$
\begin{aligned}
& \widetilde{E}(t)^{1+r / 2} \leq C_{1}(\widetilde{E}(t)-\widetilde{E}(t+1)) \\
& +\left[\frac{2}{1-12 \eta}\left(a_{0}+a_{1}\right)|\Omega|\right. \\
& \left.+\frac{2}{1-12 \eta}\left(\frac{1}{\lambda_{1}}+\frac{1}{4 \eta \lambda_{1}}\right)\|q\|^{2}\right]^{1+r / 2} .
\end{aligned}
$$

Using Nakao's Lemma 11, we conclude that

$$
\begin{aligned}
\widetilde{E}(t) \leq & \left(C_{1}^{-1} \frac{r}{2}(t-1)^{+}+\widetilde{E}(0)^{-r / 2}\right)^{-2 / r} \\
& +\frac{1}{1-12 \eta}\left(a_{0}+a_{1}\right)|\Omega| \\
& +\frac{2}{1-12 \eta}\left(\frac{1}{\lambda_{1}}+\frac{1}{4 \eta \lambda_{1}}\right)\|q\|^{2} .
\end{aligned}
$$

As $r \rightarrow \infty$, the first term of the right side of (74) goes to zero; thus, with $\widetilde{E}(t)$, we conclude

$$
\begin{aligned}
\mathbb{B} & =\left\{(u, v, \theta) \in H_{0} \mid\|\Delta u\|^{2}+\|v\|^{2}+\|\theta\|^{2}\right. \\
& \leq \frac{8}{1-12 \eta}\left(a_{0}+a_{1}\right)|\Omega| \\
& \left.+\frac{8}{1-12 \eta}\left(\frac{1}{\lambda_{1}}+\frac{1}{4 \eta \lambda_{1}}\right)\|q\|^{2}\right\}
\end{aligned}
$$

is an absorbing set for $S(t)$ in $H_{0}$.

\section{The Existence of a Global Attractor}

The main result of a global attractor reads as follows.

Theorem 14. Assume the hypotheses of Theorem 8; then the corresponding semigroup $S(t)$ of problem (8)-(11) is asymptotic compactness.

Proof. We are going to apply Lemmas 11 and 12 to prove the asymptotic smooth. Given initial data $\left(u^{0}, u^{1}, \theta^{0}\right)$ and 
$\left(v^{0}, v^{1}, \widetilde{\theta}^{0}\right)$ in a bounded invariant set $B \subset H_{0}$, let $(u, \theta),(v, \widetilde{\theta})$ be the corresponding weak solutions of problem (8)-(11). Then the differences $w=u-v, \vartheta=\theta-\widetilde{\theta}$ are the weak solutions of

$$
\begin{aligned}
& w_{t t}+\Delta^{2} w+\Delta^{2} w_{t}+v \Delta \vartheta+\Delta g \\
& =\sigma\left(\|\nabla u\|^{2}\right) \Delta u-\sigma\left(\|\nabla v\|^{2}\right) \Delta v+\Delta \phi-\Delta f_{1}, \\
& \vartheta_{t}-\Delta \vartheta+\Delta f_{2}-v \Delta w_{t}=0, \\
& w=\frac{\partial w}{\partial v}=0, \\
& \vartheta=0, \\
& w(0)=u^{0}-v^{0}, \\
& w_{t}(0)=u^{1}-v^{1}, \\
& \vartheta(0)=\theta^{0}-\tilde{\theta}_{0},
\end{aligned}
$$

where

$$
\begin{aligned}
\Delta \phi & =\phi\left(\int_{\Omega} \nabla u \nabla u_{t} d x\right) \Delta u-\phi\left(\int_{\Omega} \nabla v \nabla v_{t} d x\right) \Delta v, \\
\Delta g & =g\left(u_{t}\right)-g\left(v_{t}\right), \\
\Delta f_{1} & =f_{1}(u)-f_{1}(v), \\
\Delta f_{2} & =f_{2}(\theta)-f_{2}(\widetilde{\theta}) .
\end{aligned}
$$

Let us define

$$
E_{w}(t)=\left\|w_{t}\right\|^{2}+\|\Delta w\|^{2}+\sigma\left(\|\nabla u\|^{2}\right)\|\nabla w\|^{2}+\|\vartheta\|^{2} .
$$

As before, by density, we can assume formally that $w$ is sufficiently regular. Then, multiplying the first equation in (76) by $w_{t}$ and integrating over $\Omega$ and multiplying the second equation in (76) by $\vartheta$ and integrating over $\Omega$ and then taking the sum, we get

$$
\begin{aligned}
& \frac{1}{2} \frac{d}{d t} E_{w}(t)+\left\|\Delta w_{t}\right\|^{2}+\|\nabla \vartheta\|^{2}+\int_{\Omega} \Delta g w_{t} d x \\
& \quad+\int_{\Omega} \Delta f_{2} \vartheta d x \\
& =-\sigma^{\prime}\left(\|\nabla u\|^{2}\right)\|\nabla w\|^{2} \int_{\Omega} \Delta u u_{t} d x \\
& \quad+\Delta \sigma \int_{\Omega} \Delta v w_{t} d x+\int_{\Omega} \Delta \phi w_{t} d x-\int_{\Omega} \Delta f_{1} w_{t} d x
\end{aligned}
$$

where

$$
\Delta \sigma=\sigma\left(\|\nabla u\|^{2}\right)-\sigma\left(\|\nabla v\|^{2}\right) .
$$

Let us estimate the right hand side of (79).

Considering the continuity of $\sigma^{\prime}(\cdot)$ and estimate (39)

$$
-\sigma^{\prime}\left(\|\nabla u\|^{2}\right)\|\nabla w\|^{2} \leq C\|\nabla w\|^{2},
$$

Applying the Mean Value Theorem combined with estimate (39), by Young inequality, we get

$$
\Delta \sigma \int_{\Omega} \Delta v w_{t} d x \leq C\|\nabla w\|^{(r+2) /(r+1)}+\frac{k_{4}}{4}\left\|w_{t}\right\|_{r+2}^{r+2} .
$$

Also use the Mean Value Theorem combined with estimate (39) and Young inequality to get

$$
\begin{aligned}
& \int_{\Omega} \Delta \phi w_{t} d x=\int_{\Omega} \phi\left(\int_{\Omega} \nabla u \nabla u_{t} d x\right) \Delta w w_{t} d x \\
& -\int_{\Omega}\left[\phi\left(\int_{\Omega} \nabla u \nabla u_{t} d x\right)-\phi\left(\int_{\Omega} \nabla v \nabla v_{t} d x\right)\right] \\
& \cdot \Delta v w_{t} d x=\int_{\Omega} \phi^{\prime}\left(\xi_{2}\right) \int_{\Omega} \nabla u \nabla u_{t} d x \Delta w w_{t} d x \\
& -\int_{\Omega} \phi^{\prime}\left(\xi_{3}\right) \int_{\Omega} \nabla w \nabla w_{t} d x \Delta v w_{t} d x \leq C\|w\|\left\|\Delta w_{t}\right\| \\
& +C\|w\|\left\|\Delta w_{t}\right\|\left\|w_{t}\right\| \leq \frac{1}{4}\left\|\Delta w_{t}\right\|^{2}+C^{2}\|w\|^{2} \\
& +\frac{1}{4}\left\|\Delta w_{t}\right\|^{2}+C^{2}\|w\|^{2}\left\|w_{t}\right\|^{2} \leq \frac{1}{4}\left\|\Delta w_{t}\right\|^{2} \\
& +C^{2}\|\nabla w\|^{2}+C^{2}\|w\|^{2}\left\|w_{t}\right\|_{r+2}^{2} \leq \frac{1}{4}\left\|\Delta w_{t}\right\|^{2} \\
& +C^{2}\|\nabla w\|^{2}+\frac{C^{4}}{k_{3}}\|w\|^{2((r+2) / r)}+\frac{k_{3}}{4}\left\|w_{t}\right\|_{r+2}^{r+2},
\end{aligned}
$$

where $\xi_{2}$ is among 0 and $\int_{\Omega} \nabla u \nabla u_{t} d x$, and $\xi_{3}$ is among $\int_{\Omega} \nabla u \nabla u_{t} d x$ and $\int_{\Omega} \nabla v \nabla v_{t} d x$.

By the Holder inequality, Minkowski inequality combined with the estimate of (39), and Young inequality, we obtain

$$
\int_{\Omega} \Delta f_{1} w_{t} d x \leq C\|\nabla w\|^{(r+2) /(r+1)}+\frac{k_{4}}{4}\left\|w_{t}\right\|_{r+2}^{r+2} .
$$

On the other hand, considering assumptions (17) and (18) of $f_{2}(\cdot)$ and $g(\cdot)$,

$$
\begin{aligned}
& \int_{\Omega} \Delta f_{2} \vartheta d x \geq k_{3}\|\vartheta\|_{\varrho+2}^{\varrho+2}, \\
& \int_{\Omega} \Delta g w_{t} d x \geq k_{4}\left\|w_{t}\right\|_{r+2}^{r+2} .
\end{aligned}
$$

Thus by inserting (81)-(85) into (79), we get that

$$
\begin{aligned}
\frac{1}{2} & \frac{d}{d t} E_{w}(t)+\frac{1}{4}\left\|\Delta w_{t}\right\|^{2}+\|\nabla \vartheta\|^{2}+\frac{k_{4}}{4}\left\|w_{t}\right\|_{r+2}^{r+2} \\
& +k_{3}\|\vartheta\|_{\varrho+2}^{\varrho+2} \\
\leq & C\left(\|\nabla w\|^{2}+\|\nabla w\|^{(r+2) /(r+1)}+\|\nabla w\|^{2((r+2) / r)}\right) .
\end{aligned}
$$


Then integrating from $t$ to $t+1$ and defining an auxiliary function $F^{2}(t)$, we get

$$
\begin{aligned}
& \frac{1}{4} \int_{t}^{t+1}\left\|\Delta w_{t}\right\|^{2} d s+\int_{t}^{t+1}\|\nabla \vartheta\|^{2} d s+\frac{k_{4}}{4} \\
& \quad \cdot \int_{t}^{t+1}\left\|w_{t}\right\|_{r+2}^{r+2} d s+k_{3} \int_{t}^{t+1}\|\vartheta\|_{\varrho+2}^{\varrho+2} d s \leq E_{w}(t) \\
& \quad-E_{w}(t+1)+C \int_{t}^{t+1}\left(\|\nabla w\|^{2}+\|\nabla w\|^{(r+2) /(r+1)}\right. \\
& \left.\quad+\|\nabla w\|^{2(2(r+2) / r)}\right) d s=F(t)^{2}
\end{aligned}
$$

It is obvious that

$$
\begin{aligned}
E_{w}(t+1) & \geq E_{t}(t), \\
\frac{1}{4} \int_{t}^{t+1}\left\|\Delta w_{t}\right\|^{2} d s & \leq F(t)^{2}, \\
\int_{t}^{t+1}\|\nabla \vartheta\|^{2} d s & \leq F^{2}(t), \\
\frac{k_{4}}{4} \int_{t}^{t+1}\left\|w_{t}\right\|_{r+2}^{r+2} d s & \leq F(t)^{2}, \\
k_{3} \int_{t}^{t+1}\|\vartheta\|_{\varrho+2}^{\varrho+2} d s & \leq F(t)^{2} .
\end{aligned}
$$

Then by multiplying first equation in (76) by $w$ and integrating over $\Omega$ again, we obtain that

$$
\begin{aligned}
& \|\Delta w\|^{2}+\sigma\left(\|\nabla u\|^{2}\right)\|\nabla w\|^{2}=-\frac{d}{d t} \int_{\Omega} w_{t} w d x+\left\|w_{t}\right\|^{2} \\
& -\int_{\Omega} \Delta^{2} w_{t} w d x+\Delta \sigma \int_{\Omega} \Delta v w d x-\int_{\Omega} \Delta f_{1} w d x \\
& -\int_{\Omega} \Delta g w d x \\
& +\int_{\Omega}\left[\phi\left(\int_{\Omega} \nabla u \nabla u_{t} d x\right)-\phi\left(\int_{\Omega} \nabla v \nabla v_{t} d x\right)\right] \\
& \cdot \Delta v w d x-\phi\left(\int_{\Omega} \nabla u \nabla u_{t} d x\right)\|\nabla w\|^{2} \\
& -v \int_{\Omega} \vartheta \Delta w d x .
\end{aligned}
$$

Integrating from $t_{1}$ to $t_{2}$, we get

$$
\begin{gathered}
\int_{t_{1}}^{t_{2}}\left(\|\Delta w\|^{2}+\sigma\left(\|\nabla u\|^{2}\right)\|\nabla w\|^{2}\right) d s=\int_{\Omega} w_{t}\left(t_{2}\right) \\
\cdot w\left(t_{2}\right) d x-\int_{\Omega} w_{t}\left(t_{1}\right) w\left(t_{1}\right) d x \\
\quad+\int_{t_{1}}^{t_{2}}\left\|w_{t}\right\|^{2} d t-\int_{t_{1}}^{t_{2}} \int_{\Omega} \Delta w_{t} \Delta w d x d s
\end{gathered}
$$

$$
\begin{aligned}
& +\int_{t_{1}}^{t_{2}} \Delta \sigma \int_{\Omega} \Delta v w d x d s-\int_{t_{1}}^{t_{2}} \int_{\Omega} \Delta f_{1} w d x d s \\
& -\int_{t_{1}}^{t_{2}} \int_{\Omega} \Delta g w d x d s \\
& +\int_{t_{1}}^{t_{2}} \int_{\Omega}\left[\phi\left(\int_{\Omega} \nabla u \nabla u_{t} d x\right)-\phi\left(\int_{\Omega} \nabla v \nabla v_{t} d x\right)\right] \\
& \cdot \Delta v w d x d s-\int_{t_{1}}^{t_{2}} \phi\left(\int_{\Omega} \nabla u \nabla u_{t} d x\right)\|\nabla w\|^{2} d s \\
& +v \int_{t_{1}}^{t_{2}} \int_{\Omega} \nabla \vartheta \nabla w d x d s .
\end{aligned}
$$

Now let us estimate the right hand side of (92). Firstly, from the first inequality of (90), by holder inequality we infer that

$$
\begin{aligned}
& \int_{t}^{t+1}\left\|w_{t}\right\|^{2} d s=\int_{t}^{t+1} \int_{\Omega}\left|w_{t}\right|^{2} d x d s \\
& \quad \leq|\Omega|^{r /(r+2)}\left(\int_{t}^{t+1} \int_{\Omega}\left|w_{t}\right|^{2(r+2) / 2} d x d s\right)^{2 /(r+2)} \\
& \quad \leq C F(t)^{4 /(r+2)} ;
\end{aligned}
$$

thus there exists $t_{1} \in[t, t+1 / 4]$ and $t_{2} \in[t+3 / 4, t+1]$ such that

$$
\begin{aligned}
\left\|w_{t}\left(t_{1}\right)\right\|^{2} & \leq C F^{4 /(r+2)}(t), \\
\left\|w_{t}\left(t_{2}\right)\right\|^{2} & \leq C F^{4 /(r+2)}(t) ;
\end{aligned}
$$

then we can deduce that

$$
\begin{gathered}
\int_{\Omega} w_{t}\left(t_{2}\right) w\left(t_{2}\right) d x-\int_{\Omega} w_{t}\left(t_{1}\right) w\left(t_{1}\right) d x \\
\leq C F(t)^{4 /(r+2)}+\frac{1}{8} \sup _{t \leq \sigma \leq t+1} E_{w}(\sigma) .
\end{gathered}
$$

Use Schwarz inequality combined with the estimate of (39) and Holder inequality to obtain

$$
\begin{aligned}
& \int_{t_{1}}^{t_{2}} \int_{\Omega} \Delta w_{t} \Delta w d x d s \leq C \int_{t_{1}}^{t_{2}}\left\|\Delta w_{t}\right\| d s \\
& \quad \leq C\left(\int_{t_{1}}^{t_{2}} 1 d s\right)^{1 / 2}\left(\int_{t_{1}}^{t_{2}}\left\|\Delta w_{t}\right\|^{2} d s\right)^{1 / 2} \leq C F(t) .
\end{aligned}
$$

Apply the Mean Value Theorem combined with estimate (39) to get

$$
\int_{t_{1}}^{t_{2}} \Delta \sigma \int_{\Omega} \Delta v w d x d s \leq C \int_{t_{1}}^{t_{2}}\|\nabla w\|^{2} d s
$$

Assumption (14) of $f(\cdot)$ and the estimate of (39) imply that

$$
\int_{t_{1}}^{t_{2}} \int_{\Omega} \Delta f_{1} w d x d s \leq C \int_{t_{1}}^{t_{2}}\|\nabla w\|^{2} d s .
$$


Also from assumption (19) of $g(\cdot)$ and the estimate of (39) combined with (94), we have

$$
\begin{aligned}
\int_{t_{1}}^{t_{2}} \int_{\Omega} \Delta g w d x d s & \leq C \int_{t_{1}}^{t_{2}}\left\|w_{t}\right\|\|\Delta w\| d s \\
& \leq C F(t)^{4 /(r+2)}+\frac{1}{8} \sup _{t \leq \sigma \leq t+1} E_{w}(\sigma) .
\end{aligned}
$$

Using the Mean Value Theorem and considering the assumption of $\phi(\cdot)$ and the estimate of (39), we have

$$
\begin{gathered}
\int_{t_{1}}^{t_{2}} \int_{\Omega}\left[\phi\left(\int_{\Omega} \nabla u \nabla u_{t} d x\right)-\phi\left(\int_{\Omega} \nabla v \nabla v_{t} d x\right)\right] \\
\cdot \Delta v w d x d s=\int_{t_{1}}^{t_{2}} \phi^{\prime}\left(\xi_{4}\right) \int_{\Omega} \nabla w \nabla w_{t} d x \\
\cdot \int_{\Omega} \Delta v w d x d s \leq C \int_{t_{1}}^{t_{2}}\|w\|\left\|w_{t}\right\| d s \\
\leq C \int_{t_{1}}^{t_{2}}\|\nabla w\|^{2} d s+C \int_{t_{1}}^{t_{2}}\left\|w_{t}\right\|^{2} d s, \\
\int_{t_{1}}^{t_{2}} \phi\left(\int_{\Omega} \nabla u \nabla u_{t} d x\right)\|\nabla w\|^{2} d s \leq C \int_{t_{1}}^{t_{2}}\|\nabla w\|^{2} d s,
\end{gathered}
$$

where $\xi_{4}$ is among $\int_{\Omega} \nabla u \nabla u_{t} d x$ and $\int_{\Omega} \nabla v \nabla v_{t} d x$.

Finally, use Young inequality to get

$$
\begin{aligned}
v \int_{t_{1}}^{t_{2}} \int_{\Omega} \nabla \vartheta \nabla w d x d s \leq & \frac{v^{2}}{2} \int_{t_{1}}^{t_{2}}\|\nabla \vartheta\|^{2} \\
& +\frac{1}{2} \int_{t_{1}}^{t_{2}}\|\nabla w\|^{2} d s .
\end{aligned}
$$

By inserting (93) and (95)-(102) into (92), we obtain that

$$
\begin{gathered}
\int_{t_{1}}^{t_{2}}\left[\|\Delta w\|^{2}+\sigma\left(\|\nabla u\|^{2}\right)\|\nabla w\|^{2}\right] d s \\
\leq 3 C \int_{t_{1}}^{t_{2}}\|\nabla w\|^{2} d s+2 C \int_{t_{1}}^{t_{2}}\left\|w_{t}\right\|^{2} d s \\
+C \int_{t_{1}}^{t_{2}}\|\nabla \vartheta\|^{2} d s+2 C F(t)^{4 /(r+2)} \\
+\frac{1}{4} \sup _{t \leq \sigma \leq t+1} E_{w}(\sigma)+C F(t) .
\end{gathered}
$$

Considering (89) and (93), from (103), we have

$$
\begin{aligned}
\int_{t_{1}}^{t_{2}}\left[\|\Delta w\|^{2}+\sigma\left(\|\nabla u\|^{2}\right)\|\nabla w\|^{2}\right] d s \\
\leq 3 C \int_{t}^{t+1}\|\nabla w\| d s+C F(t)+C F^{2}(t) \\
\quad+4 C F(t)^{4 /(r+2)}+\frac{1}{4} \sup _{t \leq \sigma \leq t+1} E_{w}(\sigma) .
\end{aligned}
$$

Using Holder inequality with $1 /(\varrho+2)+(\varrho+1) /(\varrho+2)=1$,

$$
\begin{aligned}
& \int_{t_{1}}^{t_{2}}\|\vartheta\|^{2} d s \leq C \int_{t_{1}}^{t_{2}}\|\vartheta\|_{\varrho+2} d s \\
& \quad \leq C\left(\int_{t_{1}}^{t_{2}} 1^{(\varrho+2) /(\varrho+2)} d s\right)^{(\varrho+1) /(\varrho+2)} \int_{t_{1}}^{t_{2}}\|\vartheta\|_{\varrho+2}^{\varrho+2} d s \\
& \quad \leq C \int_{t_{1}}^{t_{2}}\|\vartheta\|_{\varrho+2}^{\varrho+2} d s \leq C F^{2}(t) .
\end{aligned}
$$

Then from the definition of $E_{w}(t)$ and (93), (104), and (105), we obtain that

$$
\begin{aligned}
\int_{t_{1}}^{t_{2}} E_{w}(s) d s \leq & 5 C F(t)^{4 /(r+2)}+2 C F^{2}(t)+C F(t) \\
& +\frac{1}{4} \sup _{t \leq \sigma \leq t+1} E_{w}(\sigma) \\
& +3 C \int_{t}^{t+1}\|\nabla w\| d s .
\end{aligned}
$$

For (106), by using Mean Value theorem, there exists $t^{*} \in\left[t_{1}\right.$, $t_{2}$ ] such that

$$
\begin{aligned}
E_{w}\left(t^{*}\right) \leq & 10 C F(t)^{4 /(r+2)}+2 C F(t)+4 C F^{2}(t) \\
& +\frac{1}{2} \sup _{t \leq \sigma \leq t+1} E_{w}(\sigma)+6 C \int_{t}^{t+1}\|\nabla w\| d s .
\end{aligned}
$$

From (87), we see that

$$
E_{w}(t) \leq E_{w}(t+1)+F^{2}(t) .
$$

Let $E_{w}=\sup _{t \leq \sigma \leq t+1} E_{w}(\sigma)$ with $\tau \in[t, t+1]$; then integrate (86) over $[t, \tau]$ and over $\left[t^{*}, t+1\right]$ to have

$$
\begin{aligned}
\sup _{t \leq \sigma \leq t+1} E_{w}(\sigma) \leq & E_{w}(\tau) \\
\leq & E_{w}(t+1)+F^{2}(t) \\
& \quad+C \int_{t}^{t+1}\left(\|\nabla w\|^{2}+\|\nabla w\|^{2(r+2) / r}\right) d s \\
\leq & E_{w}\left(t^{*}\right)+F^{2}(t) \\
& +C \int_{t}^{t+1}\left(\|\nabla w\|^{2}+\|\nabla w\|^{2(r+2) / r}\right) d s .
\end{aligned}
$$

Inserting (107) into (109), we obtain

$$
\begin{aligned}
\sup _{t \leq \sigma \leq t+1} E_{w}(\sigma) \leq & 10 C F(t)^{4 /(r+2)}+2 C F(t)+4 C F^{2}(t) \\
& +\frac{1}{2} \sup _{t \leq \sigma \leq t+1} E_{w}(\sigma)+6 C \int_{t}^{t+1}\|\nabla w\| d s \\
& +F^{2}(t) \\
& +C \int_{t}^{t+1}\left(\|\nabla w\|^{2}+\|\nabla w\|^{2(r+2) / r}\right) d s .
\end{aligned}
$$


Therefore from the boundary of $1+F(t)^{(1-4 /(r+2))}+$ $F(t)^{2-4 /(r+2)}$, we have

$$
\begin{aligned}
& \sup _{t \leq \sigma \leq t+1} E_{w}(\sigma) \\
& \leq C F(t)^{4 /(r+2)} \\
& \quad+C \int_{t}^{t+1}\left(\|\nabla w\|+\|\nabla w\|^{2}+\|\nabla w\|^{2(r+2) / r}\right) d s .
\end{aligned}
$$

Therefore

$$
\begin{aligned}
& \sup _{t \leq \sigma \leq t+1} E_{w}(\sigma)^{1+r / 2} \leq C\left(E_{w}(t)-E_{w}(t+1)\right) \\
& +C \sup _{0 \leq \sigma \leq T} \int_{\sigma}^{\sigma+1}\left(\|\nabla w\|+\|\nabla w\|^{2}+\|\nabla w\|^{2(r+2) / r}\right) d s .
\end{aligned}
$$

From Nakao's Lemma 11, there exists $C_{B}>0$ and $C_{T}>0$ such that

$$
\begin{gathered}
E_{w}(t) \leq C_{B}\left[(t-1)^{+}\right]^{-2 / r}+C_{T}\left(\sup _{0 \leq \sigma \leq T} \int_{\sigma}^{\sigma+1}(\|\nabla w\|\right. \\
\left.\left.+\|\nabla w\|^{2}+\|\nabla w\|^{2(r+2) / r}\right) d s\right)^{2 /(r+2)},
\end{gathered}
$$

$$
0 \leq t \leq T
$$

From the definition of $E_{w}(t)$, we have

$$
\begin{aligned}
& \left\|\left(w, w_{t}, \vartheta\right)\right\|_{H_{0}} \leq C_{B}\left[(t-1)^{+}\right]^{-2 / r} \\
& +C_{T}\left(\operatorname { s u p } _ { 0 \leq \sigma \leq T } \int _ { \sigma } ^ { \sigma + 1 } \left(\|\nabla w\|+\|\nabla w\|^{2}\right.\right. \\
& \left.\left.+\|\nabla w\|^{2(r+2) / r}\right) d s\right)^{2 /(r+2)} .
\end{aligned}
$$

Given $\varepsilon>0$, we choose $T$ large such that

$$
C_{B}\left[(t-1)^{+}\right]^{-2 / r} \leq \varepsilon,
$$

and define $\omega_{T}: H_{0} \times H_{0} \rightarrow R$ as

$$
\begin{aligned}
& \omega_{T}\left(\left(u^{0}, u^{1}, \theta^{0}\right),\left(v^{0}, v^{1}, \widetilde{\theta}^{0}\right)\right) \\
& \quad=C_{T}\left(\operatorname { s u p } \int _ { \sigma } ^ { \sigma + 1 } \left(\|\nabla w\|+\|\nabla w\|^{2}\right.\right. \\
& \left.\left.\quad+\|\nabla w\|^{2(r+2) / r}\right) d s\right)^{2 /(r+2)} .
\end{aligned}
$$

Then from (114)-(116), we get

$$
\begin{gathered}
\left\|S(T)\left(u^{0}, u^{1}, \theta^{0}\right)-S(T)\left(v^{0}, v^{1}, \tilde{\theta}^{0}\right)\right\|_{H_{0}} \\
\quad \leq \varepsilon+\omega_{T}\left(\left(u^{0}, u^{1}, \theta^{0}\right),\left(v^{0}, v^{1}, \tilde{\theta}^{0}\right)\right)
\end{gathered}
$$

for all $\left(u^{0}, u^{1}, \theta^{0}\right),\left(v^{0}, v^{1}, \widetilde{\theta}^{0}\right) \in B$.
Let $\left(u_{n}^{0}, u_{n}^{1}, \theta_{n}^{0}\right)$ be a given sequence of initial data in $B$. Then the corresponding sequence $\left(u_{n}, u_{t n}, \theta_{n}\right)$ of solutions of the problem (8)-(11) is uniformly bounded in $H_{0}$, because $B$ is bounded and positively invariant. So $\left\{u_{n}\right\}$ is bounded in $C\left([0, \infty), H_{0}^{2}(\Omega)\right) \cap C^{1}\left([0, \infty), L^{2}(\Omega)\right)$. Since $H_{0}^{2}(\Omega) \hookrightarrow$ $H_{0}^{1}(\Omega)$ compactly, there exists a subsequence $u_{n k}$ which converges strongly in $C\left([0, T], H_{0}^{1}(\Omega)\right)$. Therefore

$$
\begin{aligned}
& \lim _{k \rightarrow \infty} \lim _{l \rightarrow \infty} \int_{0}^{T}\left(\left\|\nabla u_{n k}(s)-\nabla u_{n l}(s)\right\|\right. \\
& \quad+\left\|\nabla u_{n k}(s)-\nabla u_{n l}(s)\right\|^{2} \\
& \left.\quad+\left\|\nabla u_{n k}(s)-\nabla u_{n l}(s)\right\|^{2(r+2) / r}\right) d s=0, \\
& \lim _{k \rightarrow \infty} \lim _{l \rightarrow \infty} \omega_{T}\left(\left(u_{n k}^{0}, u_{n k}^{1}, \theta_{n k}^{0}\right),\left(u_{n l}^{0}, u_{n l}^{1}, \theta_{n l}^{0}\right)\right)=0 .
\end{aligned}
$$

So $S(t)$ is asymptotically smooth in $H_{0}$. That is, Lemma 12 holds. Thus Theorem 14 is proved.

In view of Theorems 13 and 14, we have the following.

Theorem 15. The corresponding semigroup $S(t)$ of problem (8)-(11) has a compact global attractor in the phase space $H_{0}$.

\section{Competing Interests}

The authors declare that they have no competing interests.

\section{Acknowledgments}

The project is supported by the National Natural Science Foundation of China (Grant nos. 11172194 and 11401420), the Natural Science Foundation of Shanxi Province, China (Grant no. 2015011006 and Grant no. 2014011005-4).

\section{References}

[1] S. Woinowsky-Krieger, "The effect of an axial force on the vibration of hinged bars," Journal of applied Mechanics, vol. 17, pp. 35-36, 1950.

[2] J. M. Ball, "Initial-boundary value problems for an extensible beam," Journal of Mathematical Analysis and Applications, vol. 42, pp. 61-90, 1973.

[3] T. F. Ma and V. Narciso, "Global attractor for a model of extensible beam with nonlinear damping and source terms," Nonlinear Analysis. Theory, Methods \& Applications, vol. 73, no. 10, pp. 3402-3412, 2010.

[4] A. F. Pazoto and G. P. Menzala, "Uniform stabilization of a nonlinear beam model with thermal effects and nonlinear boundary dissipation," Funkcialaj Ekvacioj. Serio Internacia, vol. 43, no. 2, pp. 339-360, 2000.

[5] T. F. Ma, "Boundary stabilization for a non-linear beam on elastic bearings," Mathematical Methods in the Applied Sciences, vol. 24, no. 8, pp. 583-594, 2001.

[6] T. F. Ma, V. Narciso, and M. L. Pelicer, "Long-time behavior of a model of extensible beams with nonlinear boundary dissipations," Journal of Mathematical Analysis and Applications, vol. 396, no. 2, pp. 694-703, 2012. 
[7] Z. Yang, "On an extensible beam equation with nonlinear damping and source terms," Journal of Differential Equations, vol. 254, no. 9, pp. 3903-3927, 2013.

[8] D. Wang, J. Zhang, Y. Wang, and S. Zhang, "Attractor of beam equation with structural damping under nonlinear boundary conditions," Mathematical Problems in Engineering, vol. 2015, Article ID 857920, 10 pages, 2015.

[9] C. Giorgi, M. G. Naso, V. Pata, and M. Potomkin, "Global attractors for the extensible thermoelastic beam system," Journal of Differential Equations, vol. 246, no. 9, pp. 3496-3517, 2009.

[10] A. R. A. Barbosa and T. F. Ma, "Long-time dynamics of an extensible plate equation with thermal memory," Journal of Mathematical Analysis and Applications, vol. 416, no. 1, pp. 143165, 2014.

[11] C. Giorgi and M. G. Naso, "Modeling and steady state analysis of the extensible thermoelastic beam," Mathematical and Computer Modelling, vol. 53, no. 5-6, pp. 896-908, 2011.

[12] L. H. Fatori and T. F. Ma, "A thermoelastic system of memory type in noncylindrical domains," Applied Mathematics and Computation, vol. 200, no. 2, pp. 583-589, 2008.

[13] M. Marin, "On existence and uniqueness in thermoelasticity of micropolar bodies," Comptes Rendus de l'Académie des Sciences, Paris, Serie II, vol. 321, no. 12, pp. 475-480, 1995.

[14] M. Marin, "Some basic theorems in elastostatics of micropolar materials with voids," Journal of Computational and Applied Mathematics, vol. 70, no. 1, pp. 115-126, 1996.

[15] M. Marin and C. Marinescu, "Thermoelasticity of initially stressed bodies, asymptotic equipartition of energies," International Journal of Engineering Science, vol. 36, no. 1, pp. 73-86, 1998.

[16] M. Nakao, "Global attractors for nonlinear wave equations with nonlinear dissipative terms," Journal of Differential Equations, vol. 227, no. 1, pp. 204-229, 2006.

[17] I. Chueshov and I. Lasiecka, "Long-time behavior of second order evolution equations with nonlinear damping," Memoirs of the American Mathematical Society, vol. 195, no. 912, 2008. 


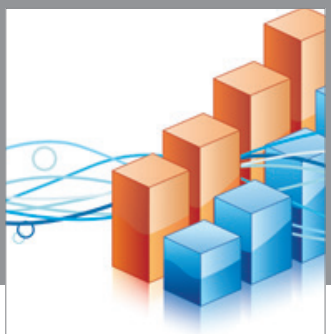

Advances in

Operations Research

vatem alat4

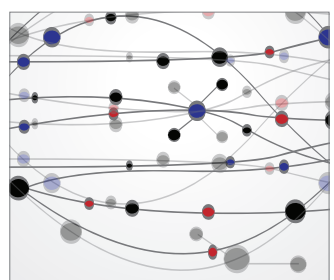

\section{The Scientific} World Journal
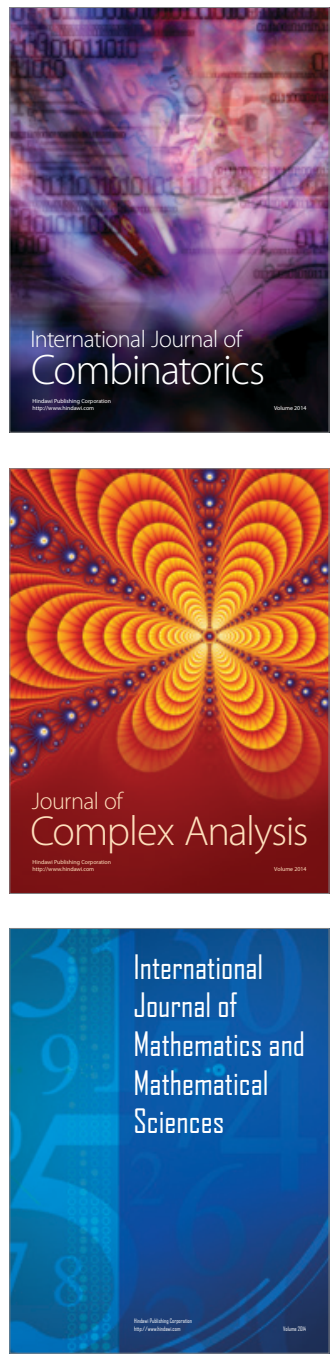
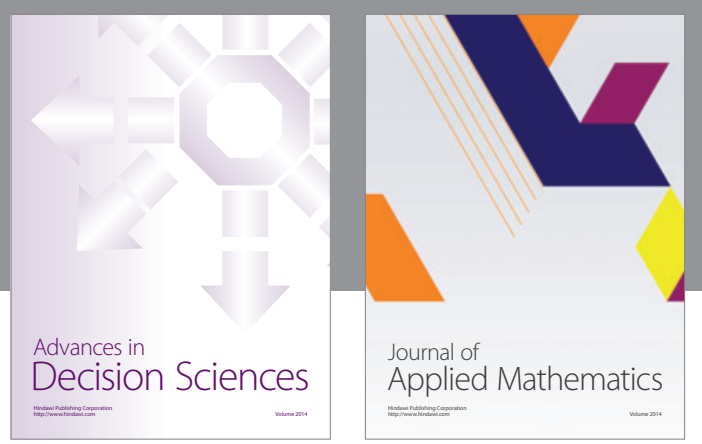

Algebra

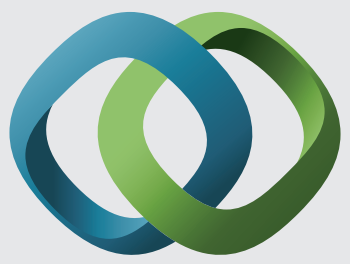

\section{Hindawi}

Submit your manuscripts at

https://www.hindawi.com
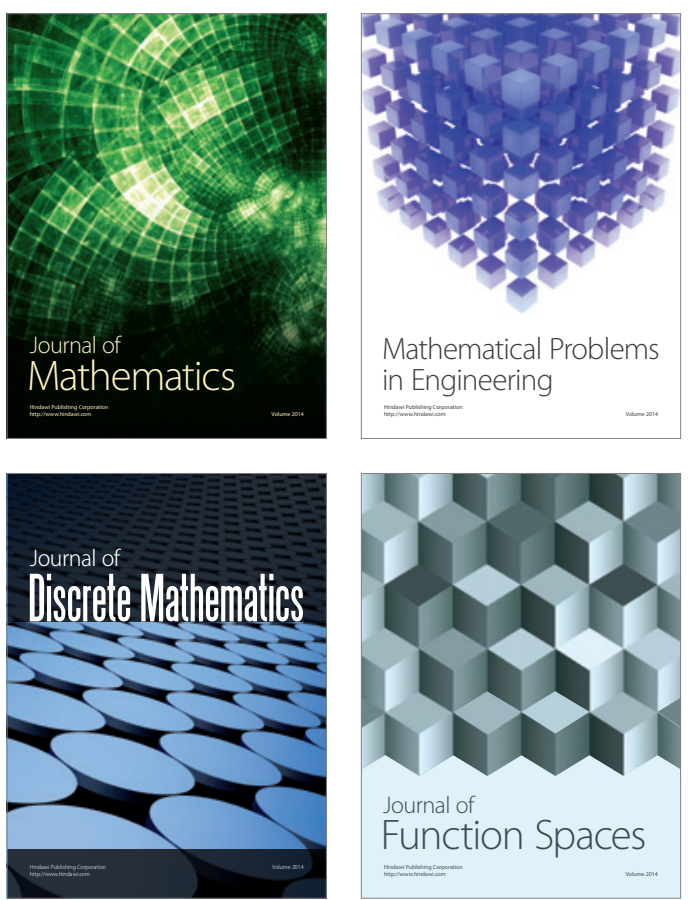

Mathematical Problems in Engineering
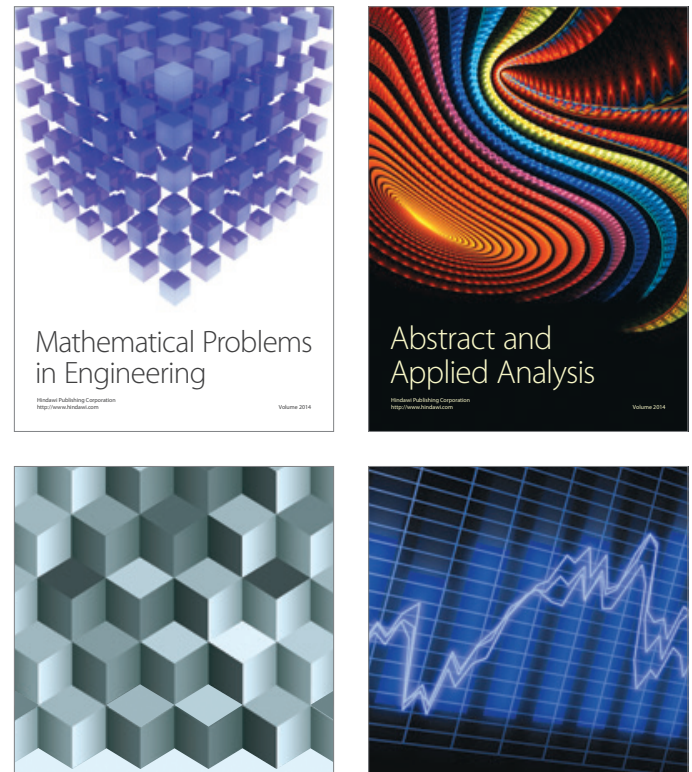

Journal of

Function Spaces

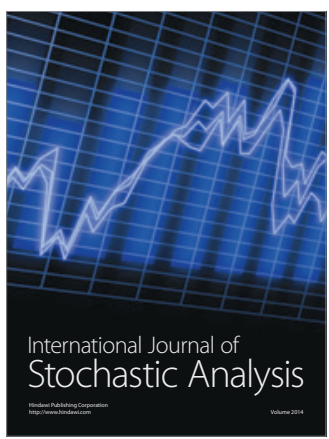

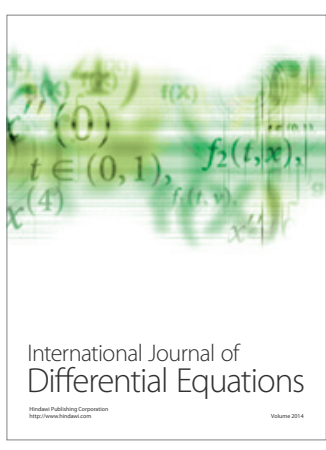
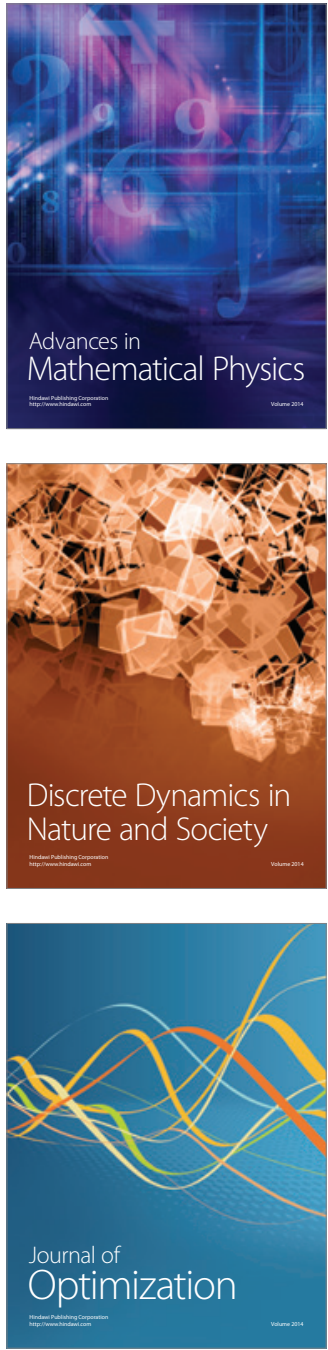